\section{Kidney \\ Blood Pressure Research}

Kidney Blood Press Res 2014;39:212-219

\begin{tabular}{l|l}
\hline DOI: $10.1159 / 000355799$ & C 2014 S. Karger AG, Basel
\end{tabular}

www.karger.com/kbr

Accepted: April 11, 2014

1423-0143/14/0393-0212\$39.50/0

This is an Open Access article licensed under the terms of the Creative Commons AttributionNonCommercial 3.0 Unported license (CC BY-NC) (www.karger.com/OA-license), applicable to the online version of the article only. Distribution permitted for non-commercial purposes only.

Review

\title{
Physical Activity and Renal Transplantation
}

\author{
Vincenzo Bellizzi ${ }^{a} \quad$ Adamasco Cupisti $^{b} \quad$ Alessandro Capitaninic Patrizia Calella ${ }^{a}$ \\ Claudia D'Alessandrob \\ a'Division of Nephrology, Dialysis and Renal Transplantation, University Hospital "San Giovanni di Dio \\ e Ruggi d'Aragona", Salerno; 'Department of Clinical and Experimental Medicine, University of Pisa, \\ Pisa; 'Nephrology and Dialysis Unit, Pescia-Pistoia Hospital, Pistoia, Italy
}

\section{Key Words}

Physical activity - Physical function - Renal transplantation - Accelerometer - METs • Cardiorespiratory fitness $\cdot$ Cardiovascular risk

\begin{abstract}
Renal transplantation is burdened by high cardiovascular risk because of increased prevalence of traditional and disease-specific cardiovascular risk factors and, consequently, patients are affected by greater morbidity and mortality. In renal transplanted patients, healthy lifestyle and physical activity are recommended to improve overall morbidity and cardiovascular outcomes. According to METs (Metabolic Equivalent Task; i.e. the amount of energy consumed while sitting at rest), physical activities are classified as sedentary (<3.0 METs), of moderate(3.0 to $5.9 \mathrm{METs}$ ) or vigorous-intensity ( $\geq 6.0 \mathrm{METs}$ ). Guidelines suggest for patients with chronic kidney disease an amount of physical activity of at least 30 minutes of moderateintensity activity five times per week (min $450 \mathrm{MET}$-minutes/week). Data on physical activity in renal transplanted patients, however, are limited and have been mainly obtained by mean of non-objective methods. Available data suggest that physical activity is low either at the start or during renal transplantation and this may be associated with poor patient and graft outcomes. Therefore, in renal transplanted patients more data on physical activity obtained with objective, accelerometer-based methods are needed. In the meanwhile, physical activity have to be considered as an essential part of the medical care for renal transplanted recipients.

Copyright (C) 2014 S. Karger AG, Basel
\end{abstract}

\section{Lifestyle, physical activity and renal transplantation}

Self-care and healthy lifestyle are recommended to kidney transplant recipients. They represents relevant aspects of the clinical care aiming to control cardiovascular risk factors and to preserve the long-term graft function. Among the component of lifestyle, physical inactivity is one of the major risk factor for mortality in the general population. However,

Dr. Vincenzo Bellizzi, MD, PhD 


\section{Kidney \\ Blood Pressure Research}

Kidney Blood Press Res 2014;39:212-219

\begin{tabular}{l|l}
\hline DOI: $10.1159 / 000355799$ & C 2014 S. Karger AG, Basel
\end{tabular}

Published onlıne: July 29, 2014

www.karger.com/kbr systematic data on physical activity in renal transplanted patients are limited. The aim of this brief review is to point out on the current level of physical activity in renal transplant patients and its relationship with either cardiovascular risk factors and nutritional parameters or clinical outcomes.

\section{Definition and measurement of physical activity}

Physical inactivity is the absence of any physical activity or exercise; conversely, physical activity is represented by any bodily movement produced by skeletal muscles requiring energy expenditure. Usual physical activity of individuals includes domestic activities, occupational doings, walking, recreational or sport activities and intentional physical exercise. The intensity of physical activity is the rate of energy expenditure necessary to perform it, the duration is the length of time spent for each session of activity, and the frequency is represented by the number of sessions of activity per week. The combination of these three components, intensity, duration and frequency, determines the whole amount of physical activity. The absolute intensity of physical activity is expressed as MET (Metabolic Equivalent Task). One MET corresponds to the amount of energy consumed by the person while sitting at rest and, conventionally, it consists in an oxygen consume of 3.5 millilitres per kilogram of body weight per minute or, alternatively, to $1 \mathrm{kCal}$ per kilogram body weight per hour. According to the intensity, physical activities are classified in moderate and vigorous. Moderate-intensity physical activities range from 3.0 to 5.9 METs, while vigorous-intensity activities are those with $\geq 6.0$ METs [1] (Table 1).

Assessment of physical activity needs reliable and accurate measurements because imprecise quantification may hidden or even mislead the relationship between physical activity and health. The most common methods used to evaluate the human physical activity are based on self-reported questionnaires which are easy to use and inexpensive, but they are also prone to several biases. Indeed, it has been reported that using the International Physical Activity Questionnaire (IPAQ), that is one of the most complete and commonly used, the relationship between physical activity and some diseases risk factors is hidden. This approach seems to underestimates the strength with disease when compared with an objective, accelerometer-based methods [2].

\section{Recommended levels of physical activity}

According to the World Health Organization, physical activity is strongly recommended for healthy individuals with the aim to improve cardiorespiratory and muscular fitness, bone 


\section{Kidney Blood Pressure Research}

and functional health, and to reduce the risk of non-communicable diseases, depression and cognitive decline [3]. For adult subjects, it is recommended at least 150 minutes of moderateintensity or, in alternative, at least 75 minutes of vigorous-intensity aerobic physical activity per week performed in sessions of 10 minutes duration, at least. To obtain additional benefits, subjects should increase moderate-intensity activity to 300 minutes or vigorous-intensity activity to 150 minutes per week. In addition, muscle-strengthening activities should be done involving major muscle groups on two or more days a week. Also subjects over 65 years with poor mobility should perform physical activity on three or more days per week. In the case they cannot do the recommended amounts of physical activity due to the health conditions, they should be as physically active as their abilities and conditions allow [3]. Benefits of being physically active implementing the WHO recommendations, compensate the possible risks associated to physical activity even at older ages. Indeed, at the recommended level of physical activity, musculoskeletal injury is uncommon. Nevertheless, to reduce the risks of musculoskeletal injuries, moderate start of physical activity and a stepwise progression to the higher levels is suggested.

The international KDOQI guidelines for patients with kidney diseases suggest patients with chronic kidney disease to undertake aerobic physical activity compatible with cardiovascular health and tolerance. It is recommended at least 30 minutes of moderateintensity physical activity five times per week (corresponding to a minimum range from 450 to 750 MET-minutes per week). This suggestion is associated with the lifestyle recommendations to achieve a healthy weight (corresponding a BMI in the range 20 to 25 $\mathrm{kg} / \mathrm{mt}^{2}$ ) and to stop smoking [4].

\section{Habitual physical function and physical activity at the start and during the renal transplantation}

Low physical functioning is expected at the time of kidney transplantation since ESRD patients often have reduced muscle mass and/or impaired physical capacity due to either co-morbidities or dialysis treatment itself. Further specific factors related to kidney transplantation itself, such as renal failure, immunosuppressive treatment, obesity, diabetes, may contribute to the impairment of physical performances in transplanted patients with respect to age- and sex-matched general population. A recent observational study evaluates the level of physical performance in a small group of 26 renal transplant candidates over 60 years (average Age, 67 yrs; Male, 58 \%; Diabetes, 65 \%; average Dialysis Vintage, 33 mos) which were compared with subjects with chronic diseases different from renal failure (diastolic heart failure; chronic obstructive pulmonary disease; high cardiovascular disease risk). Physical function was evaluated by several methods: Short Physical Performance Battery (SPPB) test, which evaluates the time needed to walk four meters at a normal pace, the time needed to stand up and sit down five times, the ability to maintain balance with feet together in fixed positions; the hand grip strength, which measures the skeletal muscle function; the Pepper Assessment Tool for Disability (PAT-D), which is a 19-item self-report questionnaire that assess difficulty with functioning. In this study, old people with advanced renal disease referred for renal transplantation had always impaired physical function as compared with elderly adults with every other chronic disease [5].

To evaluate the impact of such lower physical function in elderly renal transplant recipients on outcomes, a group of 64 old transplant recipients (average Age, 64 yrs; Male, 65 \%; Diabetes, 29 \%; average Dialysis Vintage, 26 mos) were compared with 338 younger patients (18-60 yrs), to evaluate the potential risk factors that may predict the graft and patient outcomes. Besides delayed graft function, smoking history and longer waiting time in dialysis, the lower graft and patient survival rates were associated with pre-transplant physical inactivity, too. Therefore, in elderly kidney transplant recipients the poor functional capacity predicts a poor outcome [6]. Such relationship was observed also in younger renal transplant recipients. Using the physical performance component of the Short Form 36, a 


\section{Kidney Blood Pressure Research}

retrospective study examined the physical function in a cohort of 366 dialysis patient on waiting list for renal transplantation who actually received the renal transplantation (average Age, 43 yrs; Male, 57 \%; Diabetes, 37 \%; average Dialysis Vintage, 13 mos). The Authors found that a higher pre-transplant physical functioning score was a significant predictor of lower risk for hospitalization and death in renal transplanted recipients. A limitation of this study is that the time interval between patient reporting of physical functioning status and receipt of kidney transplant was almost 10 months and, hence, this finding needs to be confirmed [7].

As a consequence of impaired physical capacity, spontaneous physical activity is expected to be low in renal transplanted patients. A small study evaluated prospectively the level of physical activity in 32 consecutive ESRD patients (average Age, 46 yrs; Male, 38 \%; Diabetes, 6 $\%$; average Dialysis Vintage, 34 mos) admitted to renal transplantation. The physical activity was evaluated by self-administered questionnaires: the Baecke questionnaire evaluated the habitual physical activities (leisure; sport; occupation) and the Five-City Project, a 7-days recall questionnaire, evaluated both the amount and the intensity of physical activity. Similarly to elderly renal transplant candidates which have reduced physical performance, also these younger renal transplanted patients (immediately prior to renal transplantation) were less physically active than healthy subjects, and their physical activity was on average $25 \%$ reduced as compared to age-matched healthy subjects [8]. Several modifiable factors may influence the level of physical activity in renal transplant recipient. A recent study evaluates the determinants of physical activity in 88 kidney recipients by mean of a semistructured interview two months after renal transplant [9]. Most of these patients were sedentary (76\%) and, besides the better physical functioning, physical activity level was also associated with private insurance and high self-efficacy. Nonetheless, such model explained only $10-15 \%$ of physical activity; younger age and higher social functioning predicted high self-efficacy for physical activity [9].

Only one small study, performed in sixteen renal transplanted children (average Age, 13 yrs, Male, 31 \%, average Dialysis Age, 6 mos; Transplant duration, 59 mos; GFR, $77 \mathrm{ml} / \mathrm{min} / 1.73 \mathrm{~m}^{2}$ ) evaluated the physical activity by an accelerometer which allows to quantify either the amount or the intensity of physical activity. Only three children $(18.5 \%)$ reached the recommended levels for moderate-vigorous physical activity and the most part of the daily individual activity (58.5\%) resulted as sedentary. Therefore, pediatric renal transplanted patients are basically physically inactive, and their physical activity level is of low intensity. In this study, as it was already observed in other studies, the exercise capacity and physical fitness were impaired, as well [10].

The behaviour of physical activity in advanced phase of renal transplantation is unclear. It is reported in a small study that patient's habitual physical activity decreases during the first month after kidney transplantation, but thereafter it increases and reaches a plateau at one year after transplantation. At this time, physical activity level is approximately 30 $\%$ higher than baseline; five years after kidney transplantation, the physical activity level seems to remain unchanged. Therefore, this study suggests that physical activity after renal transplantation may increase spontaneously [8]. Another larger, randomized clinical trial evaluated the impact of individually prescribed programs of physical exercise at the time of transplantation (97 pts; average Age, 40 yrs; Male, $56 \%$ ). One year after transplantation, the regular, habitual physical activity slightly reduced in the control group, from 47 to $36 \%$ of subjects (NS). Instead, the exercise training significantly increased from 50 to $67 \%$; nonetheless, no changes in body composition between the two groups were observed [11].

\section{Physical activity and cardio-vascular risk in renal transplantation}

The outcome of renal transplanted patients is poor as compared with the general population and the major cause of impaired survival is represented by cardiovascular diseases. These are much more frequent in transplant recipients than in the general 


\section{Kidney Blood Pressure Research}

Kidney Blood Press Res 2014;39:212-219

DOI: $10.1159 / 000355799$

Published ontIne: July 29, 2014

(c) 2014 S. Karger AG, Basel

www.karger.com/kbr

Bellizzi et al.: Physical Activity and Renal Transplantation

population because of the high prevalence of cardiovascular risk factors, both traditional and non-traditional, including inadequate lifestyle and poor physical activity. In the general population physical activity improves the CV risk profile and, thus, it is conceivable that physical activity should impact on CV risk also in renal transplanted patients. Unfortunately, data about physical activity in renal transplanted patients are limited, based on subjective self-reported questionnaire and mainly uncontrolled. Cardio-vascular risk factors related to cardiorespiratory fitness have been studied in seventy-one glucose-intolerant kidney transplant patients (average Age, 55 yrs; Male, $55 \%$; average Transplant duration, 5.7 yrs; Diabetes, $52 \%$ ). Cardiorespiratory fitness was measured by the peak oxygen uptake (VO2) by expired gas analysis and the physical activity was measured by a set of items adapted from the Physical Activity Statewide Questionnaire. The number of sessions and the time spent on different category of activity were evaluated; it was also analysed the patients' perceptions of their activeness and whether patients were achieving recommended targets of physical activity. Cardiorespiratory fitness in renal transplanted patients resulted significantly lower than expected. Specifically, patients with metabolic syndrome (63\%), which had reduced cardiorespiratory fitness, had reduced physical activity and were more frequently physically inactive (76 vs 48\%). Cardiorespiratory fitness was correlated with physical activity and the lower cardiorespiratory fitness was associated with physical inactivity. Whether increasing physical activity should improve cardiorespiratory fitness in renal transplanted patients remains to be determined [12]. This relationship was explored also in renal transplanted children. Studies evaluating the physical fitness in children with kidney transplant reported that the cardiorespiratory fitness was significantly reduced, children had low muscle strength and exercise capacity, and they were mostly physical inactive (evaluated by the Previous Day Physical Activity Recall test) $[13,14]$. Since cardiorespiratory fitness is reduced in uremic children, the impact of physical exercise was assessed in a prospective, controlled study in 16 children after renal transplantation. Physical exercise training of 3-5 hours per week allowed to increase the cardiorespiratory fitness, attaining levels comparable to controls with a sedentary lifestyle [15]. A systematic review of 21 studies (6 retrospective and 15 intervention-studies) analysed the association among exercise and several intermediate outcomes such as physical functioning, metabolic syndrome, kidney function and immune function [16]. The major evidence of the systematic review was that habitual physical activity is positively associated with quality of life and aerobic fitness and negatively associated with body fat.

\section{Physical activity and nutrition in renal transplantation}

Data about the interrelationships between physical activity and nutrition are very limited in renal transplanted patients. A prospective cohort study of 540 patients (average Age, $51 \mathrm{yrs}$; Male, $54 \%$ ) investigated the association among physical exercise and several intermediate outcomes. Physical activity was estimated by a validated questionnaires (Tecumseh Occupational Activity and Minnesota Leisure Time Physical Activity questionnaires). The level of physical activity resulted negatively related to metabolic syndrome, fasting insulin and triglycerides serum levels, and positively related to muscle mass estimated by 24-hour urinary creatinine excretion [17]. In a cross-sectional, observational study in 82 adult renal transplanted patients (average Age, 52 yrs; Male, $69 \%$; average Transplant duration, 4.4 yrs), physical activity, measured by the Physical Activity Statewide Questionnaire, was inversely related to glucose tolerance especially in females. In this study, patients with abnormal glucose tolerance test were more obese (in particular central obesity), and the higher body fat mass was associated with lower physical activity [18]. Another recent prospective study explored in 26 renal transplanted patients (average Age, 51 yrs; Male, $48 \%$ ) the role of physical activity on weight gain occurring after renal transplantation and the associated risk factors. This is the only study in adult transplanted patients in which the physical activity was objectively measured by means of an accelerometer device. One year after renal 


\section{Kidney Blood Pressure Research}

transplantation, only $19 \%$ of patients achieved to take more than 10.000 steps per day and patients with the higher weight gain, which was largely due to body fat increase, had the lower physical activity. In addition, the higher weight gain was strongly related to total cholesterol and triglyceride serum levels. Hence, fat gain after renal transplantation seems to be related to low physical activity [19]. Of course, RCTs are needed to confirm the benefits of physical activity on metabolic and nutritional status in renal transplanted patients, as well as to evaluate the potential impact on morbidity and mortality.

\section{Physical activity and outcomes in renal transplantation}

Eight-eight kidney transplant recipients (average Age, 48 yrs; Male, 58 \%; Diabetes, $9 \%$ ) were evaluated for physical activity by means of semi-structured interviews, two months after renal transplantation. It was used the Physical Activity Scale for Elderly (PASE), a self-administered, 21-item questionnaire exploring household, occupational and leisure time activities over the past 7-day period. All the patients were studied prospectively and changes in renal function over time were explored. A greater physical activity at baseline was associated with improvement in graft function in the following 6 to 12 months. These findings suggest that higher physical activity in patients with kidney transplant may favourably affect graft functioning. Whether increasing physical activity should improve renal function outcome in renal transplanted patients need to be confirmed by RCT [20]. The systematic review of 21 studies on physical activity in renal transplanted patients indicates that exercise interventions has beneficial effects also on aerobic capacity and muscle strength, but only minimal effects on metabolic syndrome, immune system and kidney function [16]. It is noteworthy that all the studies of this systematic review were based on assessments of physical activity by self-reported questionnaires rather than accelerometer devices. The association between low physical activity and cardiovascular and all-cause mortality in kidney transplant recipients was studied in a cohort of five-hundred-forty patients (average Age 51, yrs; Male, $54 \%$ ) using validated questionnaires. Along the five-year of follow-up, lower physical activity was strongly associated with increased risk for cardiovascular and all-cause mortality [17].

\section{Summary}

The investigations existing in the literature about the physical activity in renal transplanted are few and show several limitations. In most cases, the number of patients are small, lack of adequate controls, have a cross-sectional design or only a short period of follow-up. More important, virtually all adult studies estimate physical activity only with self-reported questionnaires, and therefore a subjective, inaccurate modality, rather than effectively measuring physical activity by means of objective, computable, methods. Hence, the physical activity in adult, stable renal transplant patients is ultimately largely unexplored. Given these limitations, available data suggest that patients referring for renal transplantation have impaired physical functioning, even when compared with subjects with other chronic disease. Consequently, the spontaneous physical activity is low at the time of transplantation but seems to increase, even not up to normal levels, after transplantation when physical functioning ameliorates. Moreover, low physical activity is associated with reduced cardio respiratory fitness and impaired metabolic and nutritional status. Finally, very limited data suggest the hypothesis of a link between physical activity and clinical outcomes, but no evidence exists on the impact of physical activity and exercise training on morbidity and mortality and graft survival in kidney transplanted patients (Table 2). As a whole, up to now, it seems judicious to counsel and encourage for more physical activity as a part of routine medical care in renal transplanted recipients. 


\section{Kidney \\ Blood Pressure Research}

Table 2.

Physical activity and renal Transplantation: Summary

- Renal transplant candidates have reduced physical function

- Physical function in aging renal transplant candidates is even impaired with respect to matched subjects with every other chronic disease

- Physical activity at the time of renal transplantation is reduced as compared to age-matched healthy subjects

- Pre-renal transplant poor physical functional capacity and physical inactivity are associated with worst hard outcomes during transplantation

- Physical activity is reduced during renal transplantation

- Low physical activity in renal transplanted patient is associated with impaired cardiorespiratory fitness

- Physical activity in renal transplanted patient seems inversely associated with several nutritional risk factors (metabolic syndrome, central obesity, body fat, fasting insulin, glucose tolerance, cholesterol and triglyceride concentration) and positively associated with healthier body composition (muscle mass)

- The higher physical activity after kidney transplant seems associated with a better graft functioning

- Impaired physical activity in renal transplant patients may be associated with increased risk for cardiovascular and all-cause mortality

- During renal transplantation physical activity seems to slightly increase spontaneously, even not reaching the guideline recommendations

- Barriers to physical activity in renal transplantation are largely unknown

- Exercise interventions in renal transplanted patients has positive impact on aerobic capacity and muscle strength

- Physical exercise in renal transplanted patient seems to improve but not normalize the impaired cardiorespiratory fitness

\section{Disclosure Statement}

The authors state that they do not have any conflicts and nothing to disclose.

\section{References}

1 Pate RR, Pratt M, Blair SN, Haskell WL, Macera CA, Bouchard C, Buchner D, Ettinger W, Heath GW, King AC, Kriska A, Leon AS, Marcus BH, Morris J, Paffenbarger Jr RS, Patrick K, Pollock ML, Rippe JM, Sallis J, Wilmore JH: Physical activity and public health. A recommendation from the Centers for Disease Control and Prevention and the American College of Sports Medicine. JAMA 1995;273:402-407.

2 Celis-Morales CA, Perez-Bravo F, Ibanez L, Salas C, Bailey MES, Gill JMR: Objective vs. Self-Reported Physical Activity and Sedentary Time: Effects of Measurement Method on Relationships with Risk Biomarkers. PLoS ONE 2012;7:e36345.

3 Global Recommendations on Physical Activity for Health. WHO Library Cataloguing-in-Publication Data 2010; (C) World Health Organization. ISBN 9789241599979.

4 KDIGO 2012 Clinical Practice Guideline for the Evaluation and Management of Chronic Kidney Disease. Chapter 3: Management of progression and complications of CKD. Kidney Int Suppl 2013;3:73-90.

-5 Hartmann EL, Kitzman D, Rocco M, Leng X, Klepin H, Gordon M, Rejeski J, Berry M, Kritchevsky S: Physical Function in Older Candidates for Renal Transplantation: An Impaired Population. Clin J Am Soc Nephrol 2009;4:588-594.

-6 Yango AF, Gohh RY, Monaco AP, Reinert SE, Gautam A, Dworkin LD, Morrissey PE: Excess risk of renal allograft loss and early mortality among elderly recipients is associated with poor exercise capacity. Clin Nephrol 2006;65:401-407. 


\section{Kidney \\ Blood Pressure Research}

\section{Kidney Blood Press Res 2014;39:212-219}

\begin{tabular}{l|l}
\hline DOI: 10.1159/000355799 & (c) 2014 S. Karger AG, Basel
\end{tabular}

Published onlıne: July 29, 2014

www.karger.com/kbr

7 Kutner NG, Zhang R, Bowles T, Painter P: Pretransplant Physical Functioning and Kidney Patients' Risk for Posttransplantation Hospitalization/Death: Evidence from a National Cohort. Clin J Am Soc Nephrol 2006;1:837-843.

8 Nielens H, Lejeune TM, Lalaoui A, Squifflet JP, Pirson Y, Goffin E: Increase of physical activity level after successful renal transplantation: a 5 year follow-up study. Nephrol Dial Transplant 2001;16:134-140.

-9 Gordon EJ, Prohaska TR, Gallant MP, Sehgal AR, Strogatz D, Conti D, Siminoff LA: Prevalence and Determinants of Physical Activity and Fluid Intake in Kidney Transplant Recipients. Clin Transplant 2010;24:E69-E81.

10 Clark CG, Cantell M, Crawford S, Hamiwka LA: Accelerometry-based physical activity and exercise capacity in pediatric kidney transplant patients. Pediatr Nephrol 2012;27:659-665.

11 Painter PL Hector L, Ray K, Lynes L, Dibble S, Paul SM, Tomlanovich Slascher NL: A Randomized Trial of Exercise Training after Renal Transplantation. Transplantation 2002;74:42-48.

12 Armstrong K, Rakhit D, Jeffriess L, Johnson D, Leano R, Prins J, Garske L, Marwick T, Isbel N: Cardiorespiratory Fitness Is Related to Physical Inactivity, Metabolic Risk Factors, and Atherosclerotic Burden in Glucose-Intolerant Renal Transplant Recipients. Clin J Am Soc Nephrol 2006;1:1275-1283.

13 Krasnoff JB, Mathias R, Rosenthal P, Painter PL: The Comprehensive Assessment of Physical Fitness in Children Following Kidney and Liver Transplantation. Transplantation 2006;82:211-217.

14 Painter P, Krasnoff J, Mathias R: Exercise capacity and physical fitness in pediatric dialysis and kidney transplant patients. Pediatr Nephrol 2007;22:1030-1039.

15 Lubrano R, Tancredi G, Bellelli E, Gentile I, Scateni S, Masciangelo R, De Castro G, Versacci P, Elli M: Influence of physical activity on cardiorespiratory fitness in children after renal transplantation. Nephrol Dial Transplant 2012;27:1677-1681.

16 Macdonald JH, Kirkman D, Jibani M: Kidney Transplantation: A Systematic Review of Interventional and Observational Studies of Physical Activity on Intermediate Outcomes. Adv Chronic Kidney Dis 2009;16:482-500.

-17 Zelle DM, Corpeleijn E, Stolk RP, de Greef MHG, Gans ROB, van der Heide JJH, Navis G, Bakker SJL: Low Physical Activity and Risk of Cardiovascular and All-Cause Mortality in Renal Transplant Recipients. Clin J Am Soc Nephrol 2001;6:898-905.

18 Orazio L, Hickman I, Armstrong K, Johnson D, Banks M, Isbel N: Higher Levels of Physical Activity Are Associated With a Lower Risk of Abnormal Glucose Tolerance in Renal Transplant Recipients. J Ren Nutr 2009;19:304-313.

19 Zelle DM, Kokb T, Dontjec ML, Danchelle EI, Navisa G, van Sona WJ, Bakkerf SJL, Corpeleijn E: The role of diet and physical activity in post-transplant weight gain after renal transplantation. Clin Transplant 2013;27:E484-E490.

20 Gordon EJ, Prohaska TR, Gallant MP, Sehgal AR, Strogatz D, Yucel R, Conti D, Laura A: Longitudinal analysis of physical activity, fluid intake, and graft function among kidney transplant recipients. Transpl Int 2009;22:990-998. 